\title{
Görünüm Tabanlı Yüz Tanıma Yöntemleri Kullanılarak Cinsiyet Belirleme
}

\author{
Ersin Öztürk ${ }^{1}$, Çetin Kurnaz ${ }^{2 *}$ \\ 1,2 Ondokuz Mayıs Üniversitesi, Mühendislik Fakültesi, Elektrik-Elektronik Mühendisliği Bölümü, Samsun, Türkiye \\ ( ${ }^{1}$ ORCID: 0000-0002-3841-5813, ${ }^{2}$ ORCID: 0000-0003-3436-899X)
}

(International Symposium on Multidisciplinary Studies and Innovative Technologies (ISMSIT) 2020 - 22-24 Ekim 2020)

(DOI: 10.31590/ejosat.819532)

ATIF/REFERENCE: Öztürk, E. \& Kurnaz, Ç. (2020). Görünüm Tabanlı Yüz Tanıma Yöntemleri Kullanılarak Cinsiyet Belirleme. Avrupa Bilim ve Teknoloji Dergisi, (Özel Say1), 111-120.

\section{Öz}

Teknolojik gelişmeler ile birlikte yüz ve cinsiyet tanıma sistemleri günümüzün popüler çalışmalar konusu haline gelmiştir. İnsan bilgisayar etkileşiminin temel yaklaşımlarından biri olan cinsiyet sinıflandırması, akıllı bina uygulamalarından güvenlik soruşturmalarına kadar pek çok alanda yaygın olarak kullanılmaktadır. Bu çalışmada, görünüm tabanlı cinsiyet sınıflandırma yöntemleri kullanılarak cinsiyet tespiti yapılmıştır. Görünüm tabanlı sistemlerde özellik çıkarmak için yerel ikili örüntü (LBP), Radon ve Gabor dönüşümleri kullanılmıştır. Ortaya çıkan veri matrislerindeki yüksek boyutları azaltmak için ise temel bileşen analizi (PCA) yöntemi tercih edilmiştir. Verileri sınıflandırmak için destek vektör makinesi (SVM) sınıflandırıcısı kullanılmıştır. Veri tabanı olarak FERET veri tabanındaki kişilere ait ön yüz görünümleri kullanılmıştır. Veri tabanındaki resimlerin \%70'i eğitim verisi olarak kullanıldığında \%89; \%90'1 eğitim verisi olarak kullanıldığında ise \%96'lara varan doğruluk oranlarına ulaşılmıştır. Ayıca sonuçlardan Radon dönüşümünün mevcut cinsiyet belirleme yöntemlerine dahil edilmesinin sistem doğruluğunu artırdığı görülmüştür.

Anahtar Kelimeler: Cinsiyet tanıma, Görünüm tabanlı yöntemler, LBP, PCA, SVM.

\section{Gender Classification Using Appearance Based Face Recognition Methods}

\begin{abstract}
Today, intensive studies on facial recognition systems have become an important issue. Gender classification, which is one of the basic approaches of human computer interaction, is widely used in many areas, from smart building applications to security investigations. In this study, gender recognition has been made by using appearance based gender classification systems. Local Binary Pattern (LBP), Radon and Gabor transform methods have been used to extract of features in appearance based systems. Principal Component Analysis (PCA) method has been preferred to reduce the high dimension of the resulting data matrices. Support Vector Machine (SVM) classifier is used to classify data. The front face views of the people in the FERET database were used as a database. Accuracy rates of up to $89 \%$ were achieved when $70 \%$ of the images in the database were used as training data. This value reaches up to $96 \%$ were obtained when $90 \%$ of the images were used as training data. Additionaly, the results was showed that the inclusion of Radon conversion in current gender recognition methods increases system accuracy.
\end{abstract}

Keywords: Gender recognition, Appearance based methods, LBP, PCA, and SVM.

\footnotetext{
${ }^{*}$ Sorumlu Yazar: Ondokuz Mayıs Üniversitesi, Mühendislik Fakültesi, Elektrik-Elektronik Mühendiliği Bölümü, Samsun, Türkiye, ORCID: 0000-0003-3436-899X, ckurnaz@omu.edu.tr
} 


\section{Giriş}

Günümüzde insan yüzünden cinsiyet tespit edilmesi önemli çalışma konularından biri haline gelmiştir. Özellikle teknolojinin gelişmesi, akıllı telefonların günlük hayatımızda geniş yer tutması, işlemci hızlarındaki artış ile birlikte, cinsiyet ve kimlik tespit edilmesi için hızlı ve güvenli yöntemlerin gerekliliği önemli ölçüde artmıştır. Yüzden cinsiyet ve kimlik tespit etme yöntemi, verilerin kolay elde edilmesi ve etkin bir güvenlik yöntemi olması sebebiyle biyomedikal kimlik ve cinsiyet tespiti yöntemlerinin en önemlilerinden birisidir (Alexandre, 2010; Khalifa, 2016). Yüz üzerinden cinsiyet tanıma sistemleri beş temel adımdan oluşmaktadır. Bunlar; resmin alınması (input image), ön işlemden geçirilmesi (pre-processing), özellik çıkartımı (feature extraction), boyut indirgenmesi (reduce dimension) ve sınıflandırma (classification) adımlarıdır (Dhanashri ve dig., 2016). Özellik çıkarımı bu adımların en önemlisidir (Uzun ve Gökmen, 2018). Cinsiyet sınıflandırma sistemlerinde kullanılan özellik çıkarım yöntemleri görünüm tabanlı (appearance based) ve geometrik tabanlı (geometric based) olmak üzere iki ana başlık altında toplanmaktadır (Dhanashri ve dig., 2016). Görünüm tabanlı sistemlerde özellikler yerel ikili örüntü (Local Binary Pattern, LBP), Radon, Gabor veya bu yöntemlerin birlikte kullanımları ile çıkarılmaktadır. Çıkarılan özellikler temel bileşen analizi (Principal Component Analyses, PCA) veya doğrusal ayrımcılık analizi (Linear Discriminant Analyses, LDA) gibi yöntemler kullanılarak boyutları azaltılmaktadır. Elde edilen veriler ise destek vektör makinesi (Support Vector Machine, SVM) gibi sınıflandırıcılar yardımıyla cinsiyet ayrımı sağlanmaktadır. Geometrik tabanlı sistemlerde ise amaç göz, burun, çene, ağız gibi yüz imgelerinin bulunması ve bunlar arasındaki mesafelerin oranlanması ile ortaya çıkan özelliklerin sınıflandırılmasıdır (Ramesha ve dig., 2010; Kalam ve Guttikonda, 2014; Öztürk ve Kurnaz, 2018).

Bu çalışmada, görünüm tabanlı sınıflandırma sistemleri ve FERET veri tabanı kullanılarak cinsiyet tespiti yapılmıştır. Çalışmada özellik çıkarmak için LBP, Radon ve Gabor yöntemleri; ortaya çıkan veri matrislerindeki yüksek boyutları azaltmak için ise PCA yöntemi; verileri sınıflandırmak için SVM sınıflandırıcısı kullanılmıştır. FERET veri tabanındaki resimlerinden cinsiyet tespiti üç farklı işlem adımlı yöntemle gerçeklenmiştir. İlk yöntemde cinsiyet tespiti LBP + PCA + SVM kullanılarak incelenmiş ikinci yöntemde ise Gabor + PCA + SVM kullanılmıştır. Üçüncü ve son yöntem olarak ise incelemeler Radon + Gabor + PCA + SVM kullanılarak yapılmıştır.

Makalenin geri kalanı şu şekilde organize edilmiştir: II. Bölümde Görünüm Tabanlı Yüz Tanıma Yöntemleri ve FERET ve tabanı hakkında materyal ve yöntem bilgileri sunulurken, III. Bölümde çalışmada kullanılan üç farklı yöntem için yapılan değerlendirmeler verilmiştir. Makale V. Bölüm olan Sonuçlar bölümü ile sonlandırılmıştır.

\section{Materyal ve Metot}

\subsection{Görünüm Tabanlı Yüz Tanıma Yöntemleri}

Yüz resimleri için birçok farklı yöntem kullanılarak cinsiyet tanıma sistemleri oluşturulabilir. Bu yöntemlerden sıklıkla kullanılanları yerel ikili örüntü (LBP), yerel Gabor ikili örüntü dizisi (local Gabor binary pattern sequence, LGBP), Radon dönüşümü (Radon transform, RT) ve Gabor filtresi (Gabor Filter, GF), temel bileşen analizi (PCA), destek vektör makinesi (SVM)'dir.

\subsubsection{Yerel İkili Örüntü}

Yerel ikili örüntü (LBP) en çok kullanılan yüz tanıma yöntemidir (Ahonen ve dig., 2004; Fang ve Wang, 2008). LBP ilk defa Ojala ve arkadaşları tarafindan tasarlanmıştır (Ojala ve dig., 2002). Ahonen ve arkadaşları bu yöntemi yüz tanıma sistemlerinde kullanmışlardır (Ahonen ve dig., 2004). Bu yöntemde, pikselin sekiz komşusu ile eşleştirilmesiyle görüntünün her pikseli için eşik (treshold) değeri de dikkate alınarak bir ikili sayı belirlenir. Komşu piksel merkez pikselden daha küçükse, 0 değerini alır. Aksi takdirde (yani merkez pikselden büyükse) 1 değerini alır. Görüntünün her pikseli için ikili bir sayı oluşturur. Bu ikili sayılar daha sonra görüntüyü açıklamak için kullanılır. Şekil 1, LBP yöntemine ait bir örneği göstermektedir (Ahonen ve dig., 2004). Görüntüsü örtüşmeyen alt bölgelere bölünür ve her alt bölgenin histogramını ayrı ayrı hesapladıktan sonra, görüntünün nihai özellik vektörünü oluşturmak için ağırlıklı histogramlar birleştirilir. Şekil 2'de örnek resmin öncelikle histogramı alınmıştır. Daha sonra resme LBP uygulanmış ve daha sonra yeniden histogramı alınmıştır.

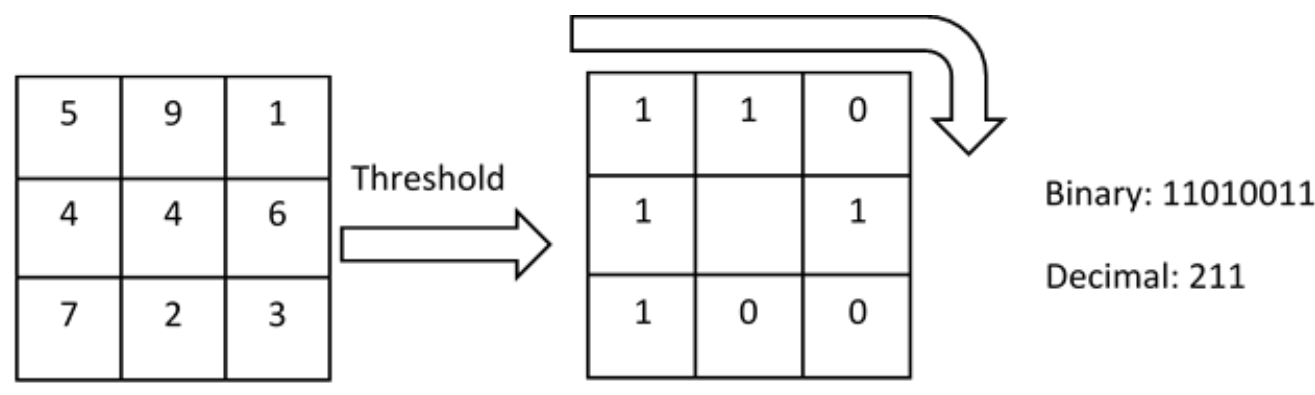

Şekil 1. LBP örneği 

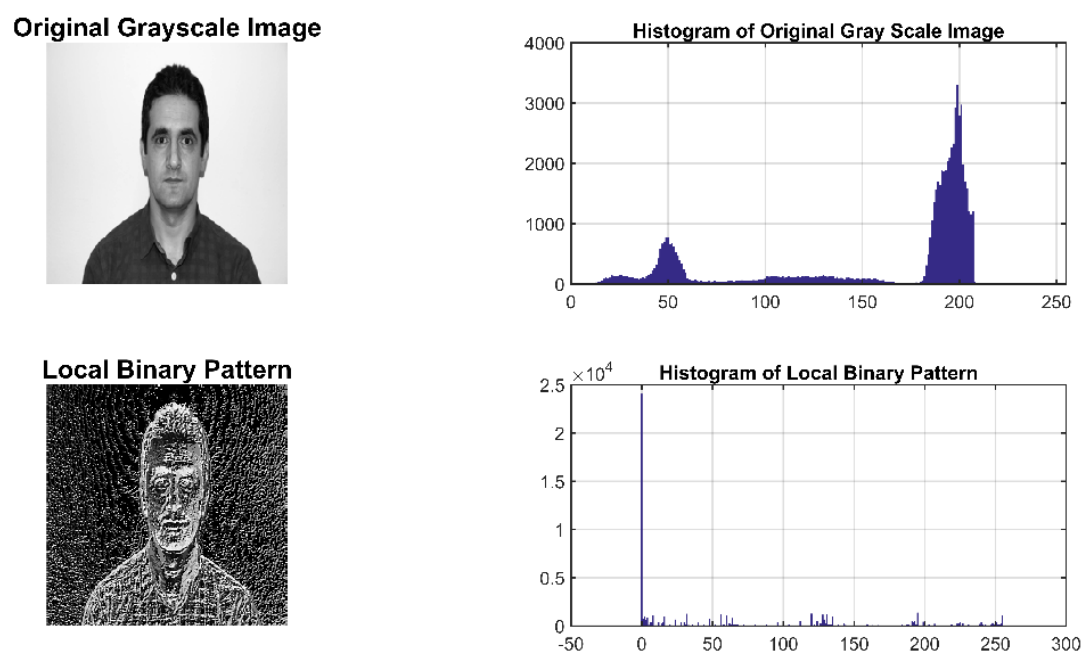

Şekil 2. Bir LBP uygulama örneği

\subsubsection{Yerel Gabor İkili Örüntü Dizisi}

Gabor filtreleri hem uzamsal hem de frekans alanında optimal lokalizasyonlarından dolayı bant geçirgen filtreler olup yönlendirme seçiciliği, doku özelliği çıkarma ve doku analizi gibi birçok uygulamada kullanılabilir (Zhang ve dig., 2002). Bir Gabor filtresi, harmonik bir fonksiyon ile Gaussian bir fonksiyonunun çarpımından oluşan lineer bir filtredir. Farklı yönelimli Gabor filtreleri hedef görüntü ile katlanarak ve bu görüntülerin ortalamaları alınarak hedef görüntüdeki farklı açı bileşenleri ortaya çıkarılabilir. Gabor filtreleri yüz tanıma sistemlerinde sıklıkla kullanılan bir yöntemdir. Özellikle özniteliklerin çıkarılmasında birçok alanda yardımcı yöntem olarak kullanılır. Bu yöntemlerden bazıları yerel Gabor ikili örüntü dizisi (LGBP) (Zang ve dig., 2005), Gabor faz örüntülerinin histogramı (histogram of Gabor phase patterns, HGPP) (Zang ve dig., 2007) ve yerel Gabor XOR örüntüleri (local Gabor XOR patterns, LGXP) (Xie ve dig., 2010) yöntemleridir. Bir görüntü, Gabor filtresi uygulandıktan sonra histogramı alınarak sınıflandırıcıya sokulabildiği gibi, Gabor filtresinden sonra LBP yapılarak da sınıflandırıcıya girdi olarak kullanılabilir. Şekil 3'de farklı çözünürlüklerdeki iki resmin Gabor çıktıları görülmektedir.
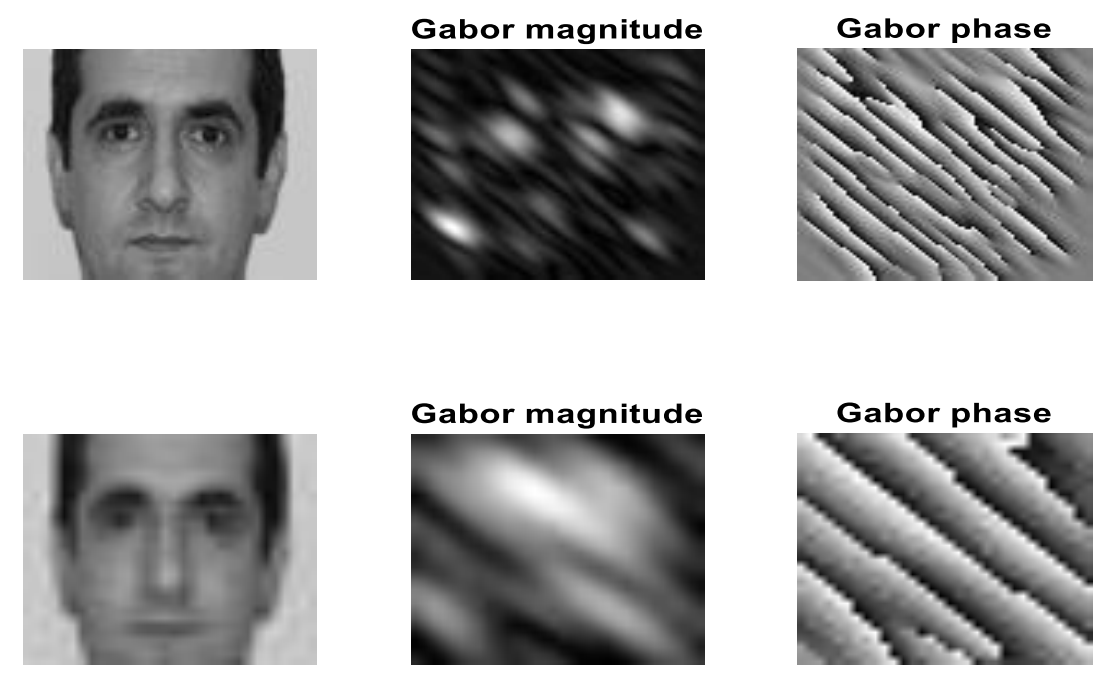

Şekil 3. Bir Gabor filtresi uygulaması

\subsubsection{Radon Dönüsümü ve Gabor Filtresi}

Radon dönüşümü, 1917'de J. Radon tarafından tanıtılan ve bir fonksiyonu alıp çeşitli yönlerde projeksiyonunu hesaplayan entegre bir dönüşümdür. Şekil 4'de f (x, y)'nin dikey doğrultudaki çizgi integrali, f (x, y)'nin x eksenine yansıtılmasıdır. Yatay yönde çizgi integrali ise y eksenine $\mathrm{f}(\mathrm{x}, \mathrm{y})$ 'nin izdüşümüdür. 


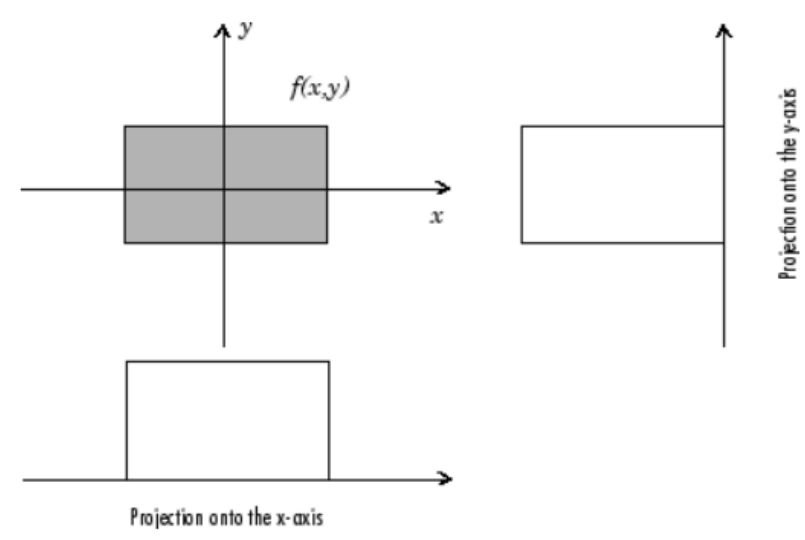

Şekil 4. Basit bir fonksiyonun yatay ve dikey eksende izdüşümü

İzdüşümler herhangi bir açı $(\theta)$ boyunca hesaplanabilir. Genel olarak, $\mathrm{f}(\mathrm{x}, \mathrm{y})$ 'nin Radon dönüşümü, Şekil 5'deki gibi y eksenine paralel $\mathrm{f}$ integrali çizgisidir.
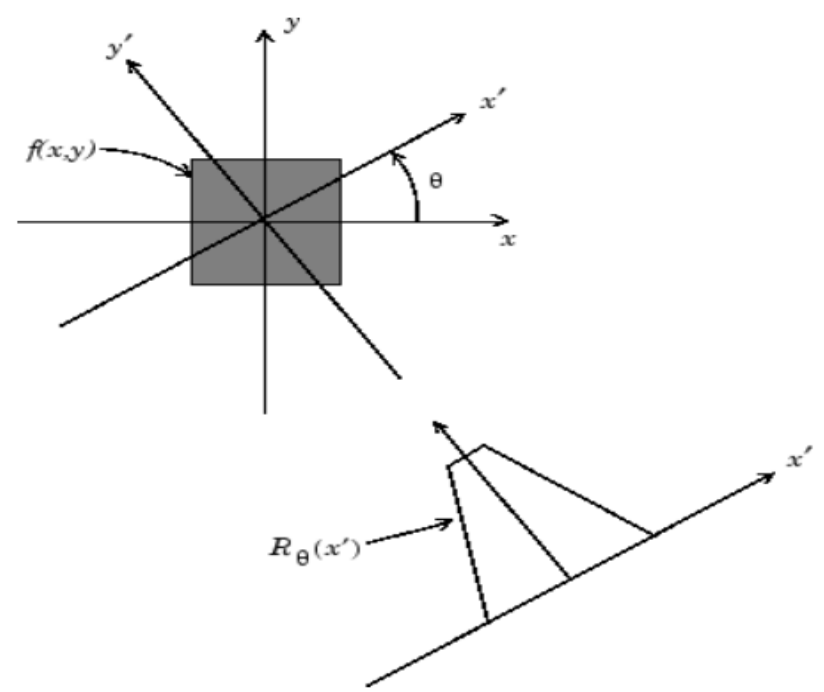

Şekil 5. Radon dönüşümünün geometrisi

Radon dönüşümü Gabor Filtresi ile kullanıldığında özellik çıkarımına pozitif etki yapmaktadır. Şekil 6'da farklı çözünürlüklerdeki iki adet resmin Radon ve Gabor dönüşümleri kullanılarak elde edilen sonuçları verilmiştir. 

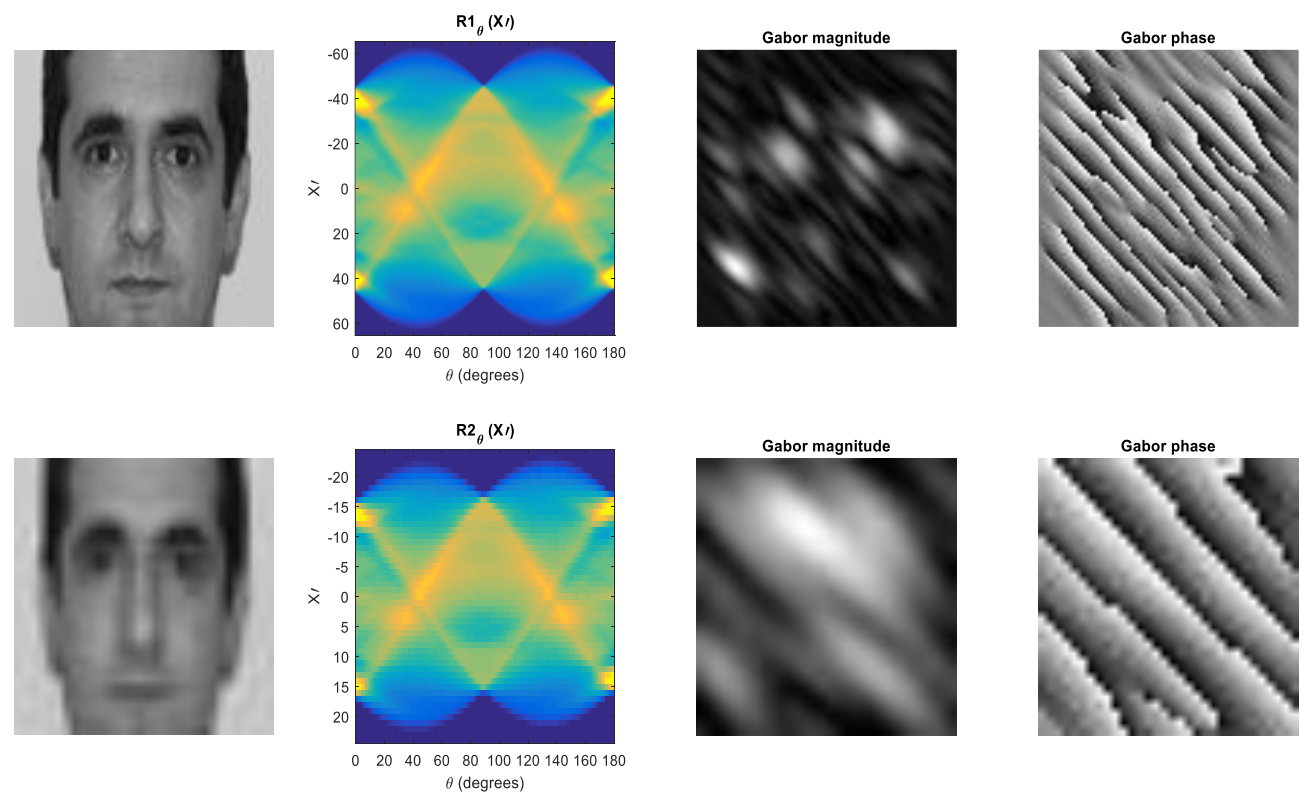

Şekil 6. Radon ve Gabor dönüşüm örnekleri

\subsubsection{Temel Bileşen Analizi}

Temel bileşen analizi (PCA), çok değişkenli bir veri seti içindeki yüksek boyutlu bir veri setinin boyutunu düşürmek için veriyi en iyi karakterize eden öznitelikleri belirleyip en az değişken ve bilgi kaybı ile ifade edebilen bir matematiksel yöntemdir. PCA, birbirleri ile doğrusal ilişkileri olan çok sayıda veriden bağımsız daha az sayıda veri elde etmek için veri setinin boyutunu düşüren ve büyük boyutlu veri setlerindeki boyutları azaltmak için kullanılan en yaygın dönüşüm tekniklerindendir. PCA'da değişken sayısı azaltılarak veri setinin boyutu küçültülebilir. PCA dönüşümü sonrasında hesaplanan değişsenler ilk değişsenlerin temel bileşenleri olarak ifade edilirler. İlk temel bileşen varyansı en büyük olan, ikinci temel bileşen ise varyansı sonraki en büyük olan olarak seçilir. Geri kalan temel bileşenler için sıralama varyans değerleri azalacak şekilde yapilır. Böylece dönüşüm sonucunda elde edilen temel bileşenler birbirlerinden bağımsız olur. Bu çalışmada PCA, resimler üzerinde özellik çıkarma işlemi yapıldıktan sonra boyut azaltmak için kullanılmıştır. Şekil 7'deki resim öncelikle kırmızı, yeşil ve mavi filtrelerden geçirilmiş ve oluşan çıktılara PCA uygulanmıştır.
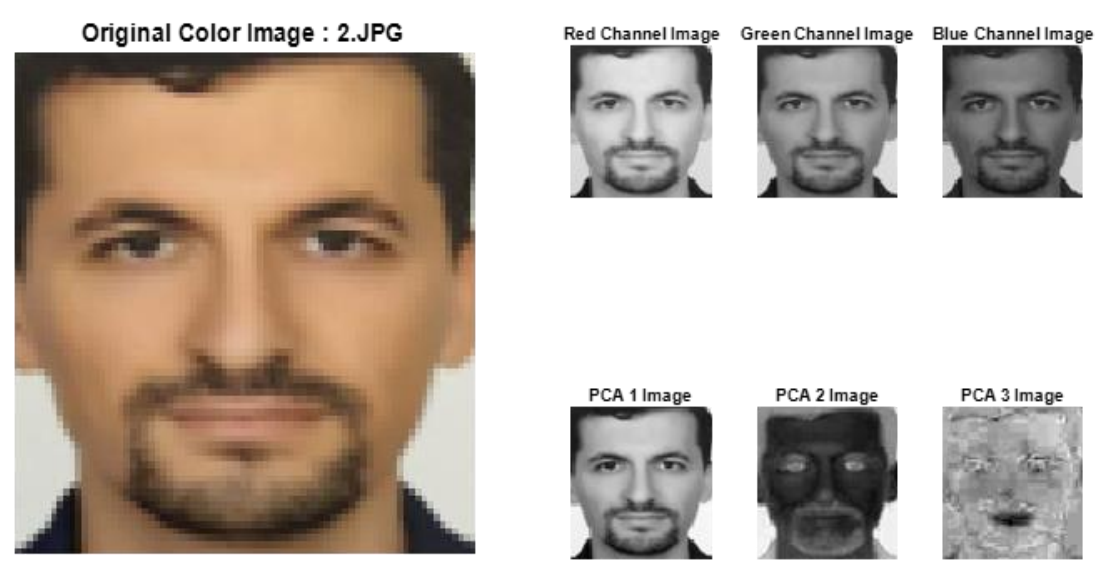

Şekil 7. PCA uygulama örneği

\subsubsection{Destek Vektör Makinesi}

Destek vektör makinesi (SVM) sınıflandırma konusunda kullanılan oldukça etkili ve basit yöntemlerden bir tanesidir. SVM istatiksel öğrenme teorisine dayalı bir gözetimli öğrenme algoritması olup temelleri 1963 yllında Vladimir Vapnik ve Alexey Chervonenkis tarafindan atılmıştır. İlk ortaya çıkışı 1960 'lı yıllar olsa da SVM, Vladir Vapnik, Berhard Boser ve Isabelle Guyon tarafından 1995 yılında geliştirilmiştir. SVM, temel olarak iki sınıfa ait verileri birbirinden bir doğru veya düzlem ile en uygun bir şekilde ayırmak amacı ile kullanılır. Bu amaca ulaşmak için karar sınırları (hiper düzlemler) belirlenir. Günümüzde destek vektör makinesi yüz tanıma sistemleri, tıbbi tahminler, zaman serisi tahminleri, ses analizleri gibi pek çok sınıflandırma probleminin çözümünde kullanılmaktadır. 


\subsection{FERET Veri Tabanı}

Yüz tanıma sistemlerinde kullanılan yöntemlerin doğruluklarının değerlendirilmesinde aynı veri tabanlarının kullanılması büyük öneme sahiptir. Aynı veri tabanlarının kullanılması ile yöntemlerin gerçek doğrulukları ortaya çıkmaktadır. Bu veri tabanlarının içinde en geniş kapsamlı ve en çok kullanılanı FERET veri tabanıdır. Bu çalışmada FERET veri tabanındaki yüzlerin ön görünümleri kullanılmıştır. Şekil 8'de FERET veri tabanından alınmış resim örnekleri verilmiştir. Literatürde veri tabanındaki kişilere ait farklı ön yüz görüntülerinden oluşan veri tabanı ile farklı yöntemler uygulanarak karşılaştırılmış ve sonuçlar Tablo 1'de verilmiştir. Tablo 1 incelendiğinde en yüksek doğrulukların FERET veri tabanı dikkate alındığında LBP ve SVM'nin birlikte kullanılması ile elde edildiği görülmektedir. Yapılan çalışmaların çoğunda sınıflandırıcı olarak genelde SVM kullanılmıştır. ANN türü sınıflandırıcıların doğruluk oranlarının SVM sınıflandırıcıya oranla daha düşük olduğu görülmektedir. Tablo 1'de verilen çalışmaların temel eksiklik noktası kullanılan eğitim/test veri setleri hakkında detaylı bilginin yer almamasıdır.
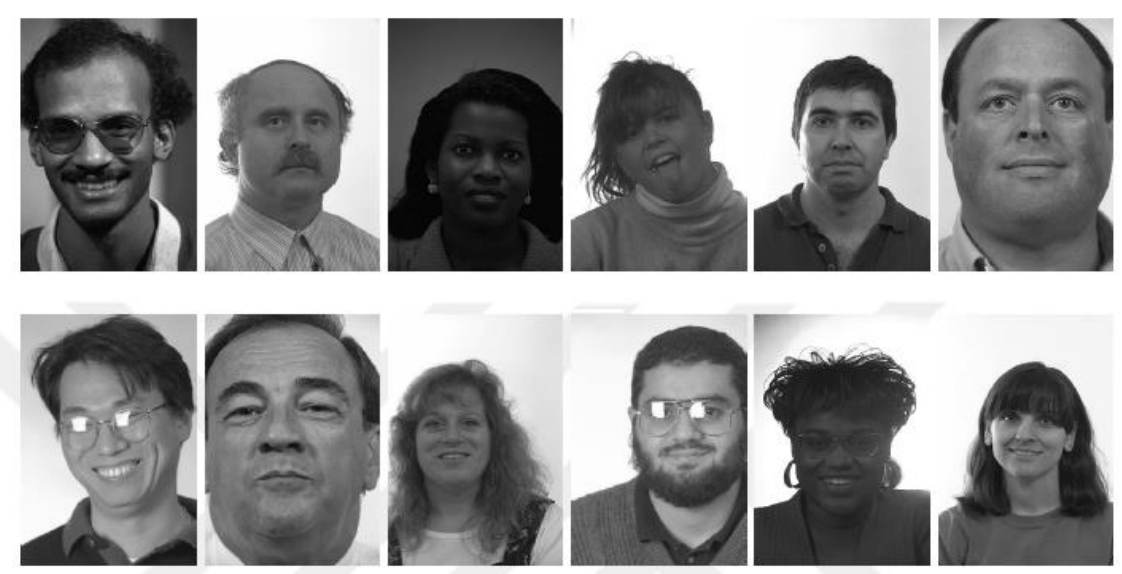

Şekil 8. FERET veri tabanında yer alan yüz resim örnekleri

Tablo 1. Yüz üzerinden cinsiyet tanıma çalışmaları

\begin{tabular}{|c|c|c|c|}
\hline Çalışma & Yöntem & Veri Tabanı & Doğruluk Oranı \\
\hline (Jain ve Huang, 2004) & ICA+LDA & FERET (sınırlı) & 99.3 \\
\hline (Iga ve dig., 2003) & GWT+SVM & HOIP (300 resim) & 97.3 \\
\hline (Zafeiriou ve dig., 2008) & RBF+SVM & XM2.VTS & 97.2 \\
\hline (Lian ve dig., 2006) & LBP+SVM & FERET & 96.75 \\
\hline (Moghaddam ve dig., 2002) & RBF+SVM & FERET & 96.62 \\
\hline (Alzarmehr ve dig., 2015) & LBP+SVM & FERET & 95.37 \\
\hline (Wang ve dig., 2012) & LCP+SVM & FERET & 95.33 \\
\hline (Lu ve Shi, 2009) & PCA+SVM & FERET & 95.30 \\
\hline (Liu ve dig., 2014) & HOG+SVM & LFW & 94.38 \\
\hline (Yang ve Ai, 2007) & LBP+Adabost & FERET & 93.3 \\
\hline (Fang ve Wang, 2008) & LBP+ANN & FERET & 92.20 \\
\hline (Fang ve Wang, 2008) & PCA+ANN & FERET & 91.91 \\
\hline (Mäkinen ve Raisamo, 2008) & LUT+ANN & FERET & 91.11 \\
\hline (Mäkinen ve Raisamo, 2008) & LBP+SVM & WEB & 78.25 \\
\hline
\end{tabular}


$\mathrm{Bu}$ çalı̧̧mada yüz üzerinden cinsiyet sınıflandırması yapmak için aşağıda verildiği gibi 3 farklı yöntem kullanılmıştır.

\author{
- Yöntem-A: LBP+PCA+SVM \\ - Yöntem-B: Gabor+PCA+SVM \\ ○ Yöntem-C: Radon+Gabor+PCA+SVM
}

\title{
3. Araştırma Sonuçları ve Tartışma
}

\subsection{Yöntem-A için Yapılan Değerlendirmeler}

Yöntem-A'da (LBF+PCA+SVM) FERET veri tabanındaki resimlere öncelikle ön işlem (preprocessing) uygulanmıştır. Bu aşamada resimler yeniden boyutlandırılmış, gri tonlara dönüştürülmüş (gray-scale) ve yüz bölgesi kesilmiştir. Daha sonra her bir resme LBP işlemi uygulanmıştır. LBP işleminden geçirilen resimler 0-255 arasında gray-scale olacak şekilde uint8 olarak kaydedilmiştir. Her resim için histogram alınmış ve histogram dağılımı veri tabanında tutulmuştur. Bu histogram dağılımları bir data matrisi oluşturacak şekilde [Resim Sayısı x 255] veri kümesi matrisi oluşturulmuştur. Bu resimlerin histogram dağılımları Şekil 9'da verilmiştir.

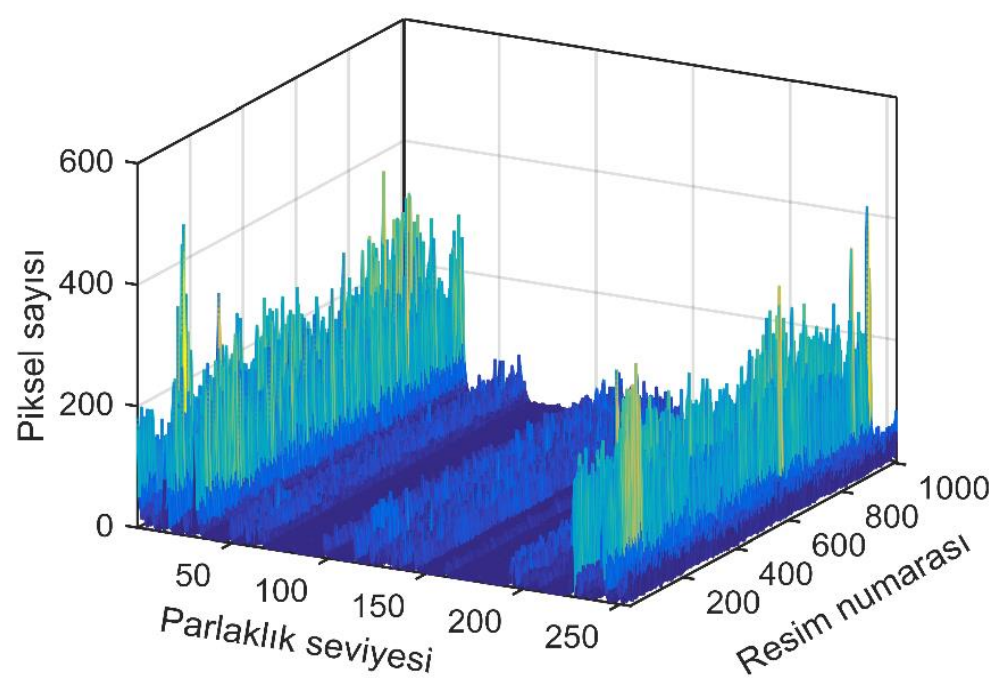

Şekil 9. Yöntem-A için LBP data matrisi

Şekil 9'daki grafiğin x ekseni 0-255 arasındaki grilik seviyelerini, y ekseni FERET veri tabanındaki her bir resmi, z ekseni piksel sayısını temsil etmektedir. LBP çıktısı ile oluşturulan grafikten de anlaşılacağı üzere bu kadar büyük bir matrisin SVM tarafından çözülmesi çok zordur. Bu nedenle boyut azaltılması (reduce dimension) gerekmektedir. PCA ile boyut azaltılması yapıldığında ortaya çıkan yeni data matrisi Şekil 10'da verildiği gibidir.

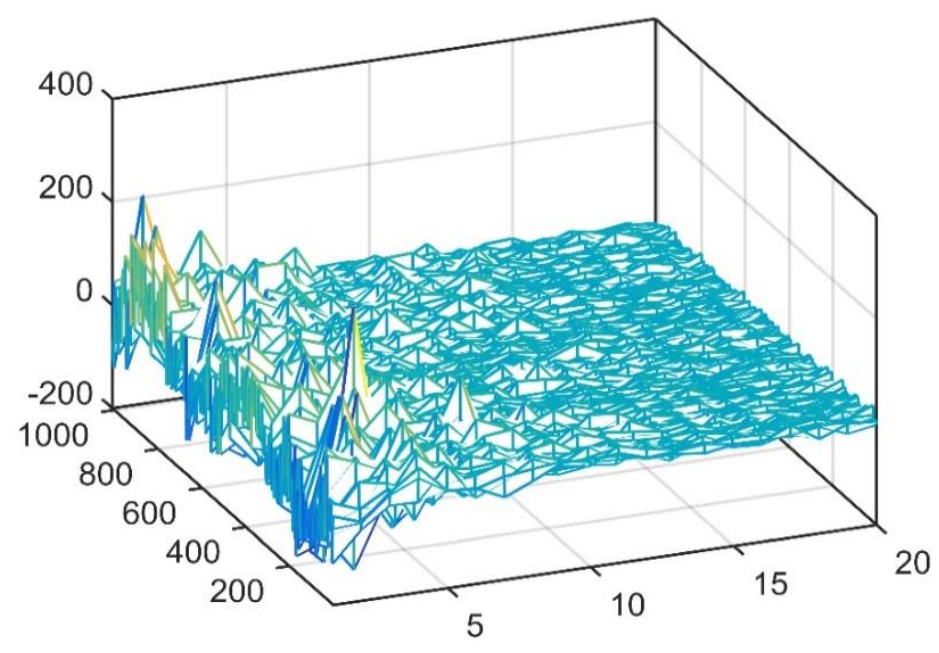

Şekil 10. Yöntem-A için PCA data matrisi 
PCA ile boyut indirgemesi yapıldığında [Resim Sayısı x 20] boyutlu bir matrise dönüştürülmüştür. Şekil 10'dan da görüldüğü gibi yapılan indirgemelerde 15. sütunda sonra anlamlı verinin olmadığı görülmektedir. PCA işlemi sonucunda indirgenmiş veri matrisi SVM sınıflandırıcısına sokulmuştur. SVM sınıflandırıcısında erkekler 1, bayanlar ise -1 değeri ile temsil edilmiştir.

\subsection{Yöntem-B için Yapılan Değerlendirmeler}

Yöntem-B'de (Gabor+PCA+SVM) FERET veri tabanındaki resimlere Yöntem-A'dakine benzer şekilde öncelikle ön işlem uygulanmıştır. Ön işlemden geçmiş veriye sonrasında Gabor filtresi uygulanmıştır. Her bir resim için histogram alınmış ve histogram dağılımı veri tabanında tutulmuştur. Bu histogram dağılımları bir data matrisi oluşturacak şekilde [Resim Sayısı x 255] veri kümesi matrisi oluşturulmuştur. Bu resimlerin histogram dağılımları grafiği Şekil 11'de verilmiştir.

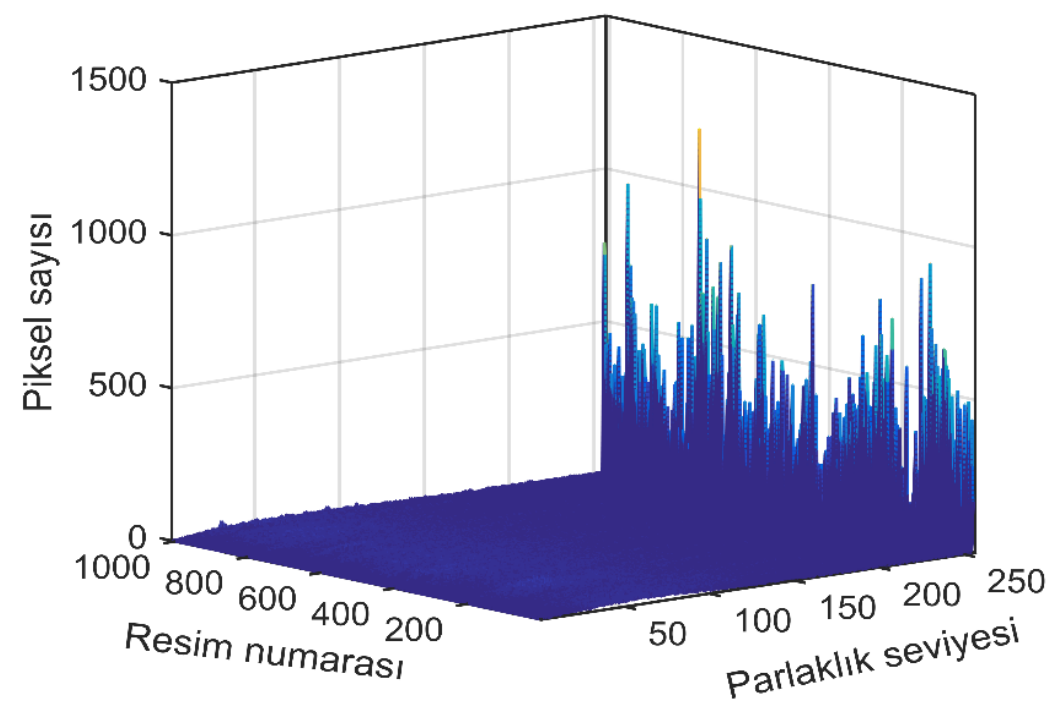

Şekil 11. Yöntem-B için data matrisi

Yöntem-A'daki gibi büyük boyutlu bu matrise PCA uygulanılarak boyut indirgemesi yapılmış ve sonuçlar Şekil 12'de verilmiştir. PCA çıktısı sonucunda oluşan indirgenmiş veri matrisi, SVM sınıflandırıcısına girdi olarak kullanılmıştır.

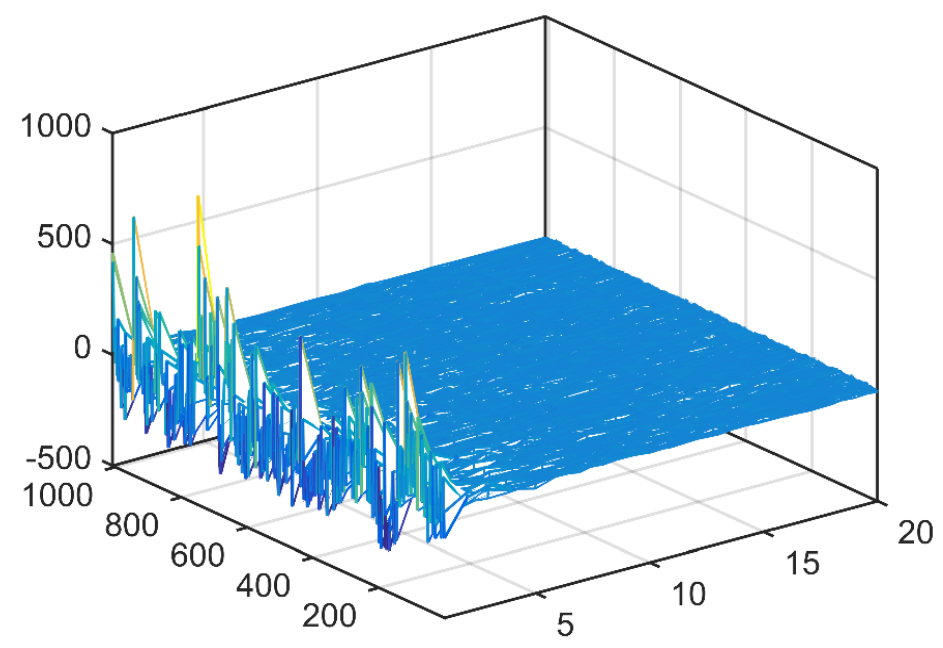

Şekil 12. Yöntem-B için PCA data matrisi 


\subsection{Yöntem-C için Yapılan Değerlendirmeler}

$\mathrm{Bu}$ yöntem (Radon+Gabor+PCA+SVM) ile Yöntem-B büyük ölçüde benzerlik göstermektedir. Yöntem-C Gabor filtresinden önce Radon dönüşümü Yöntem-B'ye eklenerek oluşturulmuştur. Radon dönüşümü cinsiyet belirleme de literatürde daha önce kullanılmamış bir yöntem olup bu çalışmada ilk kez kullanılmaktadır. Yöntem-C için elde edilen veri matrisi Şekil 13'de verilmiştir.

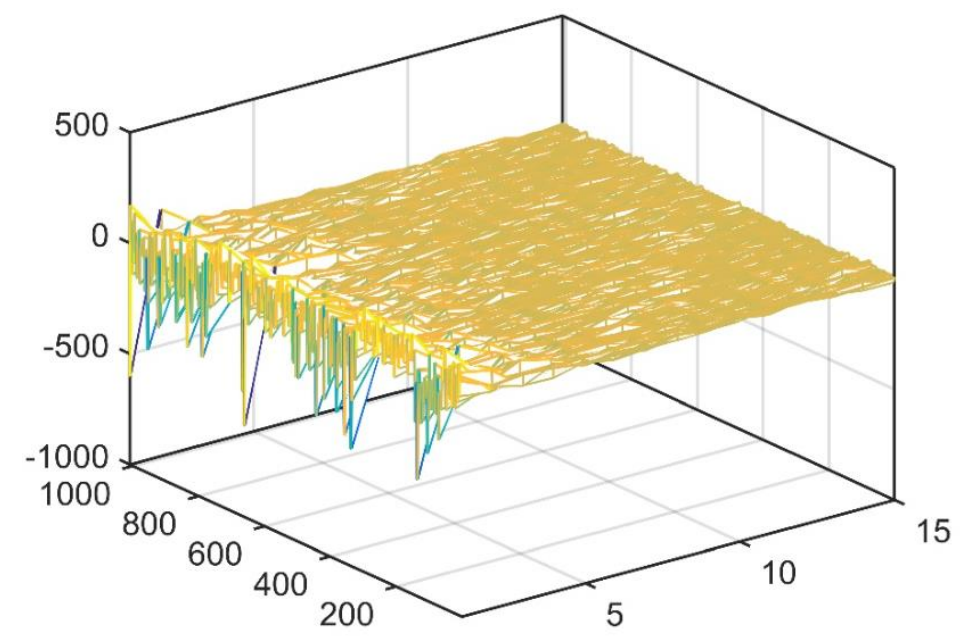

Şekil 13. Yöntem-C için PCA data matrisi

\subsection{Genel Değerlendirme ve Tartışma}

Bu çalı̧̧mada FERET veri tabanında bulunan 1010 resmin \%70'i eğitim verisi, \%30'u ise test verisi olarak kullanılmış ve Yöntem-A, Yöntem-B, Yöntem-C'nin cinsiyet sınıflandırma doğrulukları değerlendirilmiştir. Eğitim ve test verileri veri tabanından rastgele olarak seçilmiş ve bu işlem 100 defa tekrarlanarak doğruluk oranları elde edilmiştir. Sonrasında bu doğruluk oranlarının ortalamaları alınarak her bir yöntem için doğruluk oranları belirlenmiştir. Test verileri dikkate alındığında elde edilen doğruluk oranları üç yöntem için de Tablo 2'de verilmiştir. Tablo 2'den de görüldüğü gibi her üç yöntem içinde doğruluk oranları \% $89^{\prime} l a r$ civarındadır. En iyi doğruluk oranını veren yöntem \%89.42 ile Radon dönüşümünü kullanan Yöntem-C'dir. Dolayısıyla Radon dönüşümünün literatürde yer alan mevcut cinsiyet belirleme yöntemlerine dahil edilmesinin sistem doğruluğunu artırdığı görülmüsstür.

Cinsiyet tanıma sisteminin doğruluğunun seçilen eğitim ve test veri sayısı ile doğrudan ilişkili olduğu bilinmektedir. Tablo 1'de verilen çalışmalarda bu değerler açıkça belirtilmemiştir. Bu çalışmada eğitim verisi olarak toplam verinin \%70, \%80 ve \%90'1 seçilerek de değerlendirmeler yapılmıştır. Yöntem-C dikkate alındığında eğitim verisi $\% 80$ seçildiğinde doğruluk oranı $\% 94.55$ iken eğitim verisi $\% 90$ seçildiğinde bu oran \%95.98'lere kadar yükselmektedir.

Tablo 2. Benzetim sonuçları

\begin{tabular}{|c|c|l|c|}
\hline $\begin{array}{c}\text { Veri } \\
\text { Tabanı }\end{array}$ & $\begin{array}{c}\text { Resim } \\
\text { Sayısı }\end{array}$ & \multicolumn{1}{|c|}{ Yöntem } & $\begin{array}{c}\text { Doğruluk } \\
\text { Oranı }\end{array}$ \\
\hline \multirow{2}{*}{ FERET } & \multirow{2}{*}{1010} & Yöntem-A: LBP+PCA+SVM & 89.11 \\
\cline { 3 - 4 } & & Yöntem-B: Gabor+PCA+SVM & 89.02 \\
\cline { 3 - 4 } & & Yöntem-C: Radon+Gabor+PCA+SVM & 89.42 \\
\hline
\end{tabular}

\section{Sonuç}

$\mathrm{Bu}$ çalı̧̧mada yüz resimlerinden cinsiyet tanıma yöntemleri üzerine kapsamlı bir literatür çalışması yapılmıştır. FERET veri tabanı; LBP, PCA, Gabor, Radon, SVM yöntemleri kullanılarak cinsiyet tanıma doğruluk oranları incelenmiştir. Literatürde var olan çalışmalardan farklı olarak Gabor+PCA+SVM yöntemine ilave olarak Radon dönüşümü dahil edilmiş ve cinsiyet tanıma doğruluğunda bir iyileşmenin olduğu görülmüştür. FERET veri tabanı için en iyi doğruluk oranı \%89.42 ile Yöntem-C (Radon+Gabor+PCA+SVM) için elde edilmiştir. Yöntem karş̧laştırmalarında kullanılan eğitim ve test verileri sayısının sistem doğruluk oranları ile doğrudan ilişkilidir. Eğitim verisi olarak toplam verinin \%70'i yerine \%90'ı seçildiğinde doğruluk oranı \%89.42'den \%96'ya varan doğruluklara çıkabilmektedir. Bu konuda yapılacak benzer çalışmalarda bu yöntemlere ilave olarak sakal, bıyık, gözlük gibi etkenlerin ilave edilmesi ve ayrıca hareket üzerinden cinsiyet analizi çalışmalarına ağırlık verilebilir. 


\section{Kaynakça}

Ahonen, T., Hadid, A. Pietikinen, M. (2004). Face Recognition with Local Binary Patterns. The 8th European Conference on Computer Vision, 11-14 May, Prague, Czech Republic.

Alexandre, L.A. (2010). Gender recognition: A multiscale decision fusion approach. Pattern Recognition Letters, Cilt 31, Sayı 11, ss. 1422-1427.

Azarmehr, R., Laganiere, R., Lee, W.S., Xu, C., Laroche, D. (2015). Real-time embedded age and gender classification in unconstrained video. In Proceedings of the IEEE Conference on Computer Vision and Pattern Recognition Workshops, 7-12 June, Boston, USA.

Dhanashri P.L., Kailash J.K. (2016). Gender Classification using Face Image: A review. International Journal of Latest Trends in Engineering and Technology, Cilt 7, Say1 2, ss. 333-337.

Fang, Y., Wang, Z. (2008). Improving LBP features for gender classification. International Conference on Wavelet Analysis and Pattern Recognition, 30-31 August, Hong Kong, China.

Jain, A., Huang, J. (2004). Integrating independent components and linear discriminant analysis for gender classification. Sixth IEEE International Conference on Automatic Face and Gesture Recognition, 19 May, Seoul, South Korea.

Iga, R., Izumi, K., Hayashi, H., Fukano, G., Ohtani, T. (2003). A gender and age estimation system from face images. SICE 2003 Annual Conference, 4-6 August, Fukui, Japan.

Kalam, S., Guttikonda, G. (2014). Gender classification using geometric facial features. International Journal of Computer Applications, Cilt 85, Say1 7, ss. 32-37.

Khalifa, T.A.M. (2016). Predicting Age and Gender of People by Using Image Processing Techniques. Computer Engineering Atilim University, Master Thesis.

Lian, H.C., Lu, B.L. (2006). Multi-view gender classification using local binary patterns and support vector machines. The third International Conference on Advances in Neural Networks, 28 May 28 - 1 June, Chengdu, China.

Liu, H., Gao, Y., Wang, C. (2014). Gender identification in unconstrained scenarios using Self-Similarity of Gradients features. IEEE International Conference on Image Processing, 27-30 October, Paris, France.

Lu, L., Shi, P. (2009). A novel fusion-based method for expression-invariant gender classification. IEEE International Conference on Acoustics, Speech and Signal Processing, 19-24 April, Taipei, Taiwan.

Mäkinen, E., Raisamo, R. (2008). An experimental comparison of gender classification methods. Pattern Recognition Letters, Cilt 29, Sayı 10, ss. 1544-1556.

Moghaddam, B., Yang, M.H. (2002). Learning Gender with Support Faces. IEEE Transactions on Pattern Analysis and Machine Intelligene, Cilt 24, Say1 5, ss. 707-711.

Ojala, T., Pietikäinen, M., Mäenpää, T. (2002). Multiresolution Gray-Scale and Rotation Invariant Texture Classification with Local Binary Patterns. IEEE Transactions on Pattern Analysis and Machine Intelligence, Cilt 24, Say1 7, ss. 971-987.

Öztürk, E., Kurnaz, Ç. (2018). Gender Recognition System from Face Images with Artificial Neural Networks. International Eurasian Conference on Science Engineering and Technology. 22-23 November, Ankara, Turkey.

Ramesha, K., Raja, K.B., Venugopal, K.R., Patnaik, L.M. (2010). Feature Extraction-Based Face Recognition, Gender and Age Classification. International Journal of Computer Theory and Engineering, Cilt 2, Say1 5, ss. 798-820.

Uzun, M., Gökmen, M. (2018). Face Recognition with Local Walsh Transform. Signal Processing: Image Communication, Cilt 61, ss. $85-96$.

Wang, C., Huang, D., Wang, Y., Zhang, G. (2012). Facial Image-Based Gender Classification Using Local Circular Patterns. The 21st International Conference on Pattern Recognition, 11-15 November, Tsukuba, Japan.

Xie, S., Shan, S., Chen, X., Chen, J. (2010). Fusing Local Patterns of Gabor Magnitude and Phase for Face Recognition. IEEE Transactions on Image Processing, Cilt 19, Say1 5, ss. 1349-1361.

Yang, Z., Ai, H. (2007). Demographic classification with local binary patterns. The International Conference on Advances in Biometrics, 27-29 August, Seoul, Korea.

Zafeiriou, S., Tefas, A., Ioannis Pitas, I. (2008). Gender Determination Using a Support Vector Machine Variant. 16th European Signal Processing Conference, 25-29 August, Lausanne, Switzerland.

Zhang, J., Tan, T., Ma, L. (2002). Invariant Texture Segmentation via Circular Gabor Filters. The 16th IAPR International Conference on Pattern Recognition, August, Quebec, Canada.

Zhang, W., Shan, S., Gao, W., Chen, X., Zhang, H. (2005). Local Gabor Binary Pattern Histogram Sequence (LGBPHS): A Novel Non-Statistical Model for Face Representation and Recognition. The tenth IEEE International Conference on Computer Vision. 17-21 October, Beijing, China.

Zhang, B., Shan, S., Chen, X., Gao, W. (2007). Histogram of Gabor Phase Patterns (HGPP): A Novel Object Representation Approach for Face Recognition. IEEE Transactions on Image Processing, Cilt 16, Say1 1, ss. 57-68. 